\title{
Labyrinthe
}

28 | 2007 (3)

Des Juifs contre l'émancipation

\section{Du muthos au logos Le détour par la pragmatique des discours}

\section{Leopoldo Iribarren}

\section{(2) OpenEdition \\ 1 Journals}

Édition électronique

URL : http://journals.openedition.org/labyrinthe/2733

DOI : $10.4000 /$ labyrinthe.2733

ISSN : 1950-6031

\section{Éditeur}

Hermann

\section{Édition imprimée}

Date de publication : 21 décembre 2007

Pagination : 133-144

ISBN : 978-2-9526131-5-6

\section{Référence électronique}

Leopoldo Iribarren, «Du muthos au logos Le détour par la pragmatique des discours », Labyrinthe [En ligne], 28 | 2007 (3), mis en ligne le 01 octobre 2009, consulté le 19 avril 2019. URL : http:// journals.openedition.org/labyrinthe/2733; DOI : 10.4000/labyrinthe.2733

Propriété intellectuelle 


\title{
DU MUTHOS AU LOGOS \\ Le détour par la pragmatique des discours*
}

\author{
Leopoldo IRIBARREN \\ l.iribarren@mac.com
}

Quelle que soit la démarche adoptée pour rendre compte de l'émergence de la pensée rationnelle en Grèce ancienne, on est nécessairement amené à poser le problème épistémologique de la continuité et de la discontinuité en histoire ${ }^{1}$. D'autant plus lorsqu'il s'agit de déterminer les origines de la rationalité spécifiquement philosophique, dont les procédés spéculatifs et les prétentions universalisantes témoignent à la fois d'un lien et d'une rupture avec les grands récits mythiques qui portent sur la totalité du monde des dieux et des hommes. Longtemps, cette problématique a été résumée par la formule «du muthos au $\log o s »$, où le passage de l'un à l'autre supposait quelque chose comme l'avènement de la raison mettant fin aux compréhensions mythiques du monde. Conflictuelle, la formule s'écroule sous le poids des déterminations historiques des termes qui la composent. D'une part, elle s'adapte mal aux définitions du mythe que les sciences religieuses et l'anthropologie ont élaborées; de l'autre, elle est rejetée par les « démythifications » philosophiques de la raison. Bien qu'imparfaite, la formule a eu le mérite de diriger l'attention vers ce que Jean-Pierre Vernant appelait une «mutation», entre anciennes et nouvelles formes de

\footnotetext{
" Une première version de ce texte fut présentée à l'université de Lille 3, en mai 2006, dans le cadre du séminaire d'André Laks sur la philosophie présocratique. Cet échange a été d'une grande aide pour clarifier les présupposés de ma démarche. Le thème général s'inscrit dans les recherches de ma thèse, Le paradigme de la technê dans la réflexion physique grecque ancienne, sous la direction de Pierre Judet de La Combe à l'EHESS.

Dans cet article je renvoie aux fragments des auteurs présocratiques selon l'édition de référence (H. Diels et W. Kranz, Die Fragmente der Vorsokratiker, 6e éd., Berlin, 1951) dont la numérotation, accompagnée de l'abréviation usuelle (DK), est reproduite dans toutes les traductions disponibles. La traduction des textes grecs cités est mienne.

1. Cette problématique est analysée par A. Laks dans son Introduction à la «philosophie présocratique », Paris, PUF, 2006.
} 
pensée ${ }^{1}$. Du moment où une discontinuité est admise entre des paradigmes discursifs tels que la Théogonie d'Hésiode et les cosmogonies milésiennes, c'est la spécificité même de la mutation qui demande à être élucidée. On aborde habituellement cette recherche selon deux tendances historiographiques divergentes: la première est l'histoire noétique et téléologique, instaurée par Aristote et consacrée par Hegel, où l'on pose comme point de départ de la philosophie une hypothèse de connaissance; la seconde, plus historiciste et positive, cherche à situer dans le temps l'événement qui préside à une nouvelle forme de pensée dans la Grèce du VI ${ }^{\mathrm{e}}$ siècle avant J.-C.

J'aimerais ici aborder le passage du muthos au logos suivant une démarche qui s'inscrit dans l'histoire philosophique de la philosophie, sans pour autant me servir du critère de la téléologie du concept pour déterminer le début d'une discipline dont l'unité et l'autonomie sont pour le moins problématiques. En effet, l'univocité du concept s'accommode mal de l'indétermination des frontières de la philosophie présocratique, qui est liée essentiellement au foisonnement de ses objets de recherche et à la pluralité de ses démarches et de ses régimes discursifs. À l'évaluation téléologique du concept, on substituera celle de la réflexivité discursive, laquelle, en dernière instance, est une condition nécessaire du travail de conceptualisation. Le problème consiste alors à trouver un schéma permettant de rendre compte du passage de la compétence communicationnelle dont le discours du mythe fait preuve, à la compétence réflexive que l'on prête volontiers au discours philosophique.

En ce sens, deux travaux majeurs de Jean-Marc Ferry développent une pragmatique des modes de discours dont la portée, à la fois philosophique et historique, touche directement cette question ${ }^{2}$. Le discours, qui ne s'identifie pas totalement à la parole, désigne ici une «puissance de thématisation de l'expérience ${ }^{3} »$; il est cette faculté, propre à chaque culture, de mise en sens de l'expérience dans une compréhension du monde. Chez Ferry, une homologie fondamentale est posée entre l'agir

1. Jean-Pierre Vernant, «La formation de la pensée positive dans la Grèce archaïque » (1957), dans Mythe et pensée chez les Grecs. Études de psychologie historique, Paris, Maspero, 1965, p. 293.

2. Je pense notamment aux Puissances de l'expérience, Paris, Cerf, 1991 et aux Grammaires de l'intelligence, Paris, Cerf, 2004.

3. Ibidem, 1991, vol. I, p. 16 et p. 77-100. Le mot «puissance» est à prendre dans le sens que Hegel lui donne dans le Système de la vie éthique. 
et le discourir qui fait que la différenciation pragmatique des rapports au monde est consignée dans des modalités discursives également différenciées. Le discours n'est donc pas anhistorique; au contraire, il est la décantation d'une matière historique qui s'exprime chaque fois dans une modalité privilégiée. Ensuite, l'ordre d'apparition de ces modalités peut être envisagé comme une séquence idéaltypique marquée par la thématisation, à chaque étape, d'une prétention à la validité ${ }^{1}$. On distinguera ainsi quatre types dominants de discours dans les constructions symboliques consacrées: la narration, l'interprétation, l'argumentation et la reconstruction.

Ces modalités, qui doivent valoir comme types idéaux et non comme registres langagiers effectifs, se succèdent selon une dynamique réflexive. Ainsi, dans sa modalité la plus élémentaire, la narration rend compte de l'existence d'un état de choses, elle formule un procès événementiel dans des énoncés qui comportent objectivement une prétention à l'exactitude et à l'exhaustivité des observations. Elle constitue un agir réflexif en prise avec la réalité; la notion de vérité s'y entend dans le sens de la parfaite adéquation factuelle fournie par le rapport du narrateur. Cependant, le discours, en vertu de l'autonomie référentielle des symboles nominatifs, peut délibérément chercher un décalage réflexif pour thématiser une compréhension du monde qui dépasse les limites de la narration. En effet, sans qu'un indice formel vienne nécessairement le signaler, un récit peut constituer un modèle explicatif. Ainsi, au sein de la forme narrative, lorsque le sens devient autonome par rapport aux faits qui sont racontés, des implications interprétatives virtuelles apparaissent, pour lesquelles le critère de vérité ne renvoie pas à l'exactitude factuelle, mais à la congruence causale produite par l'agencement des faits - c'est là le registre de l'interprétation. La troisième étape de la montée réflexive est produite au moment où un discutant exige l'argument sous lequel des relations internes de sens, dégagées par l'acte interprétatif, deviennent possibles dans le discours

1. On entend l'expression «prétention à la validité » dans le sens que lui donne J. Habermas, à savoir comme comprenant les quatre prétentions suivantes: (I) la prétention à un sens intersubjectivement valide qui est rendu possible par la fonction du langage; (II) la prétention à la vérité, conçue comme prétention au consensus universel; (III) la prétention à la véracité; (IV) la prétention normative à la rectitude que doit contenir implicitement tout acte de parole par lequel on sollicite l'assentiment du partenaire de la communication. Voir «Was heißt Universalpragmatik?» [1976]; trad. par R. Rochlitz, dans J. Habermas, Logique des sciences sociales et autres essais, Paris, PUF, 1987. 
tenu. L'argumentation suppose en général la force de rendre problématiques les propositions; sous le principe de l'argumentation, aucun contenu de signification ne devrait être laissé dans l'ombre, hors de l'interrogation. Sans cesse révisables, et donc instables, les différentes représentations doivent soutenir face à l'autre leur propre vision du monde ${ }^{1}$. Ce schéma permet de cerner le type de prétention à la vérité dont se réclament certains textes archaïques, notamment la Théogonie d'Hésiode ( $\mathrm{VII}^{\mathrm{e}}$ siècle avant $\mathrm{J} . \mathrm{C}$. .) et les premières cosmogonies milésiennes (VI ${ }^{\mathrm{e}}$ siècle avant J.-C.), dans lesquels l'on s'accorde le plus souvent à reconnaître des manifestations, respectivement, du muthos et du logos. D'autre part, en se situant d'emblée sur le plan de l'idéalité régulatrice, le modèle d'une échelle dynamique de la réflexivité autorise à poser un point focal imaginaire où s'articulent pour la première fois les trois aspects auxquels on reconnaît la philosophie naissante, à savoir la détermination de l'origine des choses, la portée totalisante, et la prétention critiquable à la vérité.

\section{La vérité interprétée}

Quant à ses possibilités réflexives, la narration apparaît comme une modalité de discours assez limitée dans la mesure où sa prétention à la vérité relève de l'attachement descriptif à la référence. Un récit qualifié de vrai (alêthês) dans l'épopée homérique est celui qui offre une garantie de sa vraisemblance intrinsèque quand les faits qu'il raconte échappent à la perception de l'auditoire ${ }^{2}$. Il n'est donc pas étonnant que le passage réflexif de la narration à l'interprétation puisse prendre la forme d'un arrachement explicite à l'immédiateté, ce dont témoigne

\footnotetext{
1. J'ai délibérément exclu de cette ébauche le quatrième registre discursif dans le schéma de J.-M. Ferry. Il s'agit de la reconstruction et correspond à la réflexivité d'une conscience historique permettant l'ouverture aux autres identités. Foncièrement moderne, il me semble que cette forme de réflexivité n'est pas à l'œuvre en Grèce ancienne avant le mouvement sophistique.

2. Voir, par exemple, Iliade XXIII, 361; XXIV, 407. Sur l'ensemble des occurrences d'alêthês dans Homère, je renvoie à T. Krischer, «Etumos und Alêthês», Philologus, 109, 1965, p. 161-174. L'article de Krischer donne l'étymologie du mot basé sur le verbe lanthanô, «passer inaperçu », «échapper à la conscience» et rend donc alêthes par «ce qui n'échappe pas à l'attention». Malgré l'évidence homérique, l'interprétation heideggérienne (Sein und Zeit, p. 32 sqq. et 219 sqq.) construite à partir de lêthê, «oubli», qui fait de l'alêtheia quelque chose comme le dévoilement d'une dimension méconnue du réel (avec tous les développements ontologiques qui suivent), a frayé son chemin, notamment dans le champ de l'anthropologie comparée.
} 
l'adresse des Muses de l'Olympe au berger Hésiode alors que paissaient ses agneaux au pied de l'Hélicon:

Bergers des champs, mauvaises ignominies, rien que des ventres, nous savons dire de nombreux mensonges semblables aux réalités (etuma), mais nous savons aussi, lorsque nous le voulons, proclamer des vérités (alêthea).

(Théogonie, 26-28).

Dans un essai remarquable ${ }^{1}$, O. Gigon a voulu à juste titre marquer avec ces paroles le début de la philosophie, en soulignant qu'Hésiode se retourne contre Homère pour dire quelque chose de totalement nouveau. Selon cette interprétation, Hésiode reçoit l'ordre de chanter le vrai et non des fictions qui ont l'apparence de réalités; le monde du mensonge vraisemblable serait donc celui d'Homère, auquel Hésiode oppose une vérité proprement philosophique, c'est-à-dire portant sur les origines et la totalité des choses. Or, en se servant de l'hexamètre dactylique, Hésiode aurait mis en contradiction ses moyens d'expression avec ses intentions philosophiques. Mais y a-t-il vraiment une contradiction, comme le pense Gigon, entre le genre traditionnel où s'inscrit un poème comme la Théogonie et un discours portant les marques de la philosophie? Plutôt qu'une contradiction entre épopée et philosophie, on soutiendra, avec Heinz Wismann², que face à la forme générique qui est la sienne, Hésiode ménage dans le proème de la Théogonie l'ouverture réflexive qui confère à son discours le statut d'œuvre originelle prétendant à un type de vérité radicalement nouveau, tout en restant indissociable d'une situation énonciative traditionnelle.

Si les Muses disent - avec tout le poids d'une intervention divine au style direct - savoir chanter aussi bien le vrai que le faux, c'est bien ce double pouvoir constitutif de la poésie qu'elles inspirent, qui demande à être élucidé. Or, comment relier les paroles mensongères ( $p s e u d e a$ polla legein) et la disposition à proférer des vérités (alêthea gêrusasthai)?

1. Der Ursprung der griechischen Philosophie. Von Hesiod bis Parmenides, Bâle, Schwabe \& Co., 1945.

2. «Propositions pour une lecture d'Hésiode», dans F. Blaise et P. Judet de La Combe (éds.), Le Métier du Mythe. Lectures d'Hésiode, Villeneuve d'Ascq, Presses universitaires du Septentrion, 1996, p. 15-24. 
Comment la connaissance peut-elle s'articuler avec le faux? En scrutant ces vers de plus près, on s'aperçoit que les faussetés dont il est ici question sont le produit de l'imitation de choses sensibles (etuma) et non pas une fabulation sans référent dans le réel. Quand etumon qualifie un discours, les occurrences homériques montrent qu'il s'agit d'une parole dont le référent est présent, ou dont la confirmation effective est sur le point de se produire'. Suivant l'emploi homérique, le vers 27 de la Théogonie présente le pseudos comme un énoncé faux, mais semblable au dénotatif; le mensonge d'Hésiode se définit par rapport au réel et non par opposition au discours vrai, les alêthea qui sont proférés par les Muses restent une option qui n'est donc pas incompatible avec les mensonges dits. Or, si les Muses mentent, c'est non pas par défaut de perception, comme il arrive parfois à certains personnages d'Homère ${ }^{2}$, mais dans l'intention avouée de construire une fiction.

Un discours qualifié d'etumon assume pleinement la fonction dénotative des choses présentes ${ }^{3}$, tandis qu'un discours alêthês se pose d'emblée comme la configuration d'une succession de faits qui échappent à l'expérience de l'auditeur. Entre les deux types de certitude revendiqués par l'épopée homérique, les Muses d'Hésiode font surgir un registre de la parole qui, dans l'agencement de mensonges, produit une totalité signifiante se prévalant d'une prétention à la vérité. Le discours du mythe savant qu'elles vont proférer se définit par rapport à ce qui n'est pas du mythe, il se démarque explicitement du discours référentiel ${ }^{4}$. Le proème de la Théogonie thématise une référence descriptive fictionnelle qui, dans le mouvement même de sa narration, fait émerger une nouvelle pertinence au niveau du sens. Mais le vrai se manifeste dans un récit contenant de nombreux mensonges à condition que l'auditeur tienne à l'écart le dessein dénotatif. La variante introduite par Hésiode (Théogonie 28) de la formule «dire le vrai» (alêthea muthêsasthai), employée par la poésie épique ${ }^{5}$, vient renforcer cette interpréta-

1. Voir Iliade XVIII, 128; Odyssée XXIII, 26-27 et l'emploi formulaire en I, 174; IV, 645; V, 232; XIV, 186; XXIV, 258, 297, 403.

2. Voir Iliade X, 534; Odyssée IV, 140.

3. Les composés de eteos expriment la réalité et l'authenticité. Voir T. Krischer, loc. cit., p. 166-67. Rappelons en passant que chez Démocrite, etumos désigne le registre du réel dans son authenticité originaire, les atomes et le vide, par opposition au conventionnel, les qualités sensibles.

4. Je dois cette remarque à $\mathrm{P}$. Judet de La Combe, dont le séminaire à l'EHESS porte sur la prétention à la vérité des poétiques grecques anciennes.

5. Voir Iliade VI, 382; Odyssée XIV, 125; XVII, 15; XVIII, 342. 
tion: la vérité du chant ne relève pas du dire (legein) comme les mensonges tirés de références réelles (etuma), mais d'une modalité particulière de la parole divine, "proférer» (gêrusasthai), qui se distingue du dire quotidien des mortels. Dire des choses fausses (pseudea legein) est donc la condition de la révélation de vérités (alêthea gêrusasthai). Les Muses annoncent aux auditeurs du poème la perspective selon laquelle une fonction référentielle plus dissimulée, une vérité intellectuelle accessible à la seule écoute avertie, peut se dégager du récit. Alors que les faits racontés dans la narration sont à la portée de tous, le déploiement des significations vraies demande à l'interprète de passer par-delà les références dans le but de dégager de la structure du récit la compréhension universalisante de l'événement. Comme le signale Paul Ricœur, «ce sera la tâche de l'interprétation de déployer la visée d'un monde libérée, par suspension, de la référence descriptive ${ }^{1} \gg$.

Le passage à l'interprétation comme mode discursif réflexif à l'égard de la narration engage l'aède et l'auditeur dans une entente implicite. Le don des Muses à Hésiode consiste en une narration où la récurrence d'épisodes mensongers - tels les relations érotiques, les engendrements, les conflits entre les puissances, les partages et les ruses - véhiculent les significations vraies qui rendent compte de l'ordre du monde présent. La compréhension de la totalité des êtres prend le détour d'un récit qui déploie, dans la régularité de ses connexions internes, la dialectique opposant la fécondité infinie qui assure le devenir du monde à la limitation et réglementation d'une telle prolifération.

\section{La vérité argumentée}

L'apparition d'une modalité discursive affranchie des conditions d'énonciation du vers épique pour consigner des systèmes cosmologiques dont l'ambition totalisante - comprenant une histoire générale de l'univers depuis les origines jusqu'à la fin - prolonge celle des théogonies, entraîne des gains et des pertes quant aux possibilités réflexives et analytiques qu'il convient d'examiner. D'une part, en renonçant aux ressources de l'énonciation poétique, la prose se prive des moyens 
propres à déployer les virtualités interprétatives auxquelles une œuvre comme la Théogonie délègue l'élaboration du sens. D'autre part, la prose ne sollicite pas le concours ni l'autorité des Muses pour son énonciation, elle n'est pas non plus l'objet d'une performance; au lieu d'interprétants, elle présuppose l'institution du discutant. La prose profite donc à un échange horizontal, mais, en contrepartie, les certitudes du discours cessent d'être protégées par une garantie d'ordre divin, elles deviennent soudainement vulnérables, tandis qu'un poème comme la Théogonie, tout en requérant les compétences de l'interprète, n'appelle pas lui-même de contestation. La prose entraîne la responsabilité individuelle quant aux prétentions à la vérité ${ }^{1}$ : elle impose à l'auteur l'obligation de défendre sa vision du monde face au lecteur ou éventuel discutant, en même temps que les certitudes acquises cessent d'être protégées contre une mise en question. La perte du «préjugé d'autorité », qui découle de l'abandon des conditions formelles d'énonciation de la poésie inspirée, instaure une vérité argumentative: il ne s'agit pas de déployer un monde de significations vraies, mais d'expliquer et de justifier ses propos. Le poème se contente de suggérer sa rationalité, le traité en prose est censé argumenter la sienne.

Nulle part, la faculté de la prose à rendre problématiques les propositions qu'elle exprime n'est aussi évidente que dans les premières cosmologies grecques. Dans la succession qui va de Thalès à Anaximène en passant par Anaximandre, la prose se constitue en véhicule privilégié d'un discours argumentatif qui cherche à poser des principes universels pour fonder la vérité d'une compréhension du monde ${ }^{2}$. Ouverts par leur forme même à la contestation, ces systèmes se répondent et se succèdent dialectiquement à l'intérieur de ce qu'on pourrait appeler, rétrospectivement, un espace autonome des raisons. Les représentations antagonistes postulant soit un principe déterminé, soit un principe indifférencié, sont obligées de justifier, ne serait-ce que pour elles-

1. Le cas d'Hécatée de Milet (Jacoby, FgrH 1. F. 1) est à cet égard paradigmatique. Sur l'émergence de la responsabilité épistémologique de l'auteur, voir G.E.R. Lloyd, Adversaries and Authorities, Cambridge, CUP, 1997; A. Laks, «Écriture, prose, et les débuts de la philosophie ancienne», Methodos, 1, 2001, p. 131-151.

2. On ne sait pas si Thalès a écrit son propos sur l'eau comme matière originaire de l'univers. Mais le point essentiel, c'est qu'en postulant un principe concret au lieu d'une divinité (comme le Chaos chez Hésiode ou Acousilaos) il a suscité des répliques en prose : de la part d'Anaximandre, une «exposition sommaire des opinions» (Diogène Laërce II, 1) et de la part d'Anaximène, un écrit «simple et dépouillé en langue ionienne» (Diogène Laërce II, 3). 
mêmes, leur propre cohérence logique. En même temps, ce mouvement argumentatif immanent à la prose relativise de facto la prétention à la validité des propositions exprimées. Toujours reçue comme une tentative de justification, l'argumentation s'expose naturellement à la déstabilisation criticiste ${ }^{1}$.

Selon la pragmatique des registres discursifs que nous schématisons à présent, l'espace autonome des raisons suppose la stabilisation d'un objet de référence intentionnelle vers lequel convergent les divers points de vue; il s'agit d'un thème commun permettant aux perspectives de se rejoindre et d'amorcer un accord sur les propositions qui visent à établir des vérités à son endroit. Dans cette histoire, le rôle de stabilisateur de l'objet de la recherche revient naturellement à Thalès. C'est d'ailleurs lui qu'Aristote désigne en Métaphysique A, 3, comme le fondateur (archêgos) d'un type nouveau de philosophie, en rupture avec le thème des généalogies divines. Certes, Thalès n'est pas un penseur systématique, cela n'empêche pas qu'aux yeux d'Aristote l'affirmation concernant l'eau comme principe originaire des choses fait surgir une manière spécifiquement philosophique de considérer le monde, dans la mesure où ce postulat rompt avec les représentations mythologiques $^{2}$. Or, si dans la perspective d'Aristote, Thalès apparaît comme le premier penseur archaïque à vaincre les résistances sémantiques posées par le discours mythique aux tentatives de régularisation du monde sous un principe explicatif unique, dans la perspective qui est la nôtre, l'affirmation de Thalès opère la délimitation d'un horizon idéal des affirmations au-delà des situations énonciatives de la poésie inspirée.

Cependant, dans une histoire de la philosophie ancienne envisagée comme une montée dynamique de la réflexivité discursive, Thalès reste une figure de transition entre l'interprétation universalisante des grands mythes et l'argumentation d'une compréhension du monde. S'il est vrai que son postulat fige l'objet de référence qui permettra aux diverses perspectives théoriques de se rejoindre, il néglige de justifier le contenu énoncé. C'est avec Anaximandre que se réalise le passage logique d'un mode de connaître aux modes d'expliquer et de justifier

1. Voir à ce propos, J.-M. Ferry, 1991, t. I, p. 122-128.

2. Sur la construction historiographique qui fait de Thalès le point de départ d'un mouvement téléologique aboutissant à la philosophie péripatéticienne, voir J. Mansfeld, «Aristotle and others on Thales, or the beginnings of natural philosophy», Mnemosyne, 38, 1985, p. 109-129. 
relevant de l'intelligence critique ${ }^{1}$. Le caractère paradigmatique de son écrit, fondateur du genre de traités Sur la nature, instaure un schéma argumentatif où le seul principe posé doit être en mesure de rendre compte de tous les phénomènes naturels, de la naissance du monde jusqu'à son dépérissement, en passant par les corps célestes, les manifestations météorologiques et l'apparition du vivant ${ }^{2}$. Faute d'argumentation, la simple affirmation de Thalès appelle aussitôt une contestation $^{3}$. Pour Anaximandre, la réponse que Thalès apporte à la question du principe du devenir reste déterminée de façon trop concrète et particulière pour pouvoir s'égaler à la forme la plus générale du problème. À l'eau, il oppose alors l'apeiron, une substance en mouvement perpétuel, qualitative et quantitativement indéfinie. Sans entrer dans les complexités physiques et ontologiques du système d'Anaximandre, il convient de porter attention à la manière dont s'instaure par rapport à Thalès une prétention argumentative à la vérité. Selon la célèbre citation rapportée par Simplicius, le devenir s'explique selon un schème de contrariété symétrique où les puissances élémentaires entrent en rapports alternatifs de génération et de dissolution arbitrés par le temps: «selon la nécessité (kata to khreôn), car elles se rendent justice et châtiment de leur injustice les unes aux autres selon l'ordre du temps» (DK 12 B1). Exprimée dans la forme d'une loi universelle, la phrase d'Anaximandre ne se contente pas d'énoncer ce qui advient à partir de l'apeiron, mais elle explique et justifie le principe même qui fonde le processus au cours duquel la loi du devenir fait justice ${ }^{4}$.

Dans l'histoire de la philosophie ancienne, la figure du discutant - qu'il soit disciple, auditeur ou compagnon - symbolise le rapport dialectique entre les différents systèmes qui se succèdent dans une dynamique de déstabilisation criticiste. Avec Anaximène, auditeur

1. Sur la logique pragmatique qui sous-tend le passage d'une modalité à l'autre, voir J.-M. Ferry, 2004, p. 137-56.

2. Partant d'une analyse du mode de présentation de la doxographie, Ch. Kahn (Anaximander and the Origins of Greek Cosmology, New York, Columbia U. P., 1960, p. 199-213) montre que le traité d'Anaximandre fournit le modèle remarquablement stable des traités Sur la nature (peri phuseos).

3. Le type de lien qui unit Anaximandre à Thalès varie selon le doxographe: «successeur et élève» pour Simplicius (DK 12 A 9), «auditeur» pour Hippolyte (DK 12 A 11), «compagnon» pour Plutarque (DK 12 A 10). Dans tous les cas, on suppose l'instance d'un discutant.

4. Sur le vocabulaire et la forme judiciaire du fragment d'Anaximandre, voir M. M. Sassi, «Anassimandro e la scrittura della 'legge' cosmica», dans M. M. Sassi (éd.), La Costruzione del discorso filosofico nell'età dei Presocratici, Edizioni della Normale, Pise, 2006, p. 3-26. 
d'Anaximandre, selon la tradition, le traité en prose atteint la plénitude de ses compétences réflexives. Ce n'est pas que le contenu de son système soit promis à la permanence dans le paysage intellectuel ionien: on sait qu'il sera rapidement mis en cause par Héraclite. Mais dans la série paradigmatique que forment les trois philosophes milésiens, le traité d'Anaximène est le seul dont nous soit parvenue une thématisation de sa prétention à la vérité. En effet, dans le résumé d'Hippolyte (DK 13 A7 §1), dont on peut supposer qu'il s'agit d'une paraphrase assez fidèle du traité d'Anaximène, on s'aperçoit que la formule choisie par le Milésien pour mettre en avant la validité universelle de sa doctrine comporte des échos qui évoquent le savoir intégral de l'épopée: «Il disait que le principe premier est l'air illimité, dont provient ce qui devient (ta ginomena), ce qui est devenu (ta gegonota), ce qui existera (ta esomena) ainsi que les dieux et les choses divines, tandis que le reste provient des descendants de celui-ci.» Anaximène légitime son entreprise savante en s'appropriant la formule par laquelle Homère qualifie le savoir du devin Calchas dans l'Iliade (I, 70), qui porte sur «ce qui est, ce qui sera et ce fut». Déjà Hésiode, dans la Théogonie (32 et 38), l'avait détournée pour désigner l'objet du poème dans son déploiement temporel. La formule porte donc en elle tout le poids d'une situation énonciative inspirée, validant par là l'entreprise totalisante de la poésie. Or, dans ce nouveau contexte, elle vient plutôt sceller le décalage réflexif introduit par le traité en prose. Il s'agit d'assumer et en même temps de dépasser l'héritage d'un certain type de prétention à la vérité. D'une part, en employant la formule, Anaximène inscrit la démarche intellectuelle milésienne dans la tradition d'un discours qui porte sur l'origine des choses et qui embrasse les trois dimensions du temps. De l'autre, le fait de l'inscrire dans un traité en prose - prenant soin en outre de l'adapter à cet objet de recherche en constante transformation qu'est la phusis ${ }^{1}$ - a pour conséquence de placer la prétention à la vérité de ses contenus dans une situation communicationnelle ouverte à la déstabilisation. Anaximène s'installe au rang de sujet épistémologique vis-à-vis du monde, son système lui permet de déduire les événements passés et futurs, mais son discours n'est aucunement protégé contre une mise en question.

1. En substituant justement le verbe «devenir» (gignomai) au verbe «être» (eimi). 
S'il est impossible de tracer dans la réalité historique une frontière nettement définie entre le muthos et le logos, leur examen sous la forme de modalités discursives idéaltypiques, définies par leur puissance réflexive, permet de saisir certains effets spécifiques du passage de l'un à l'autre. Envisagée comme moteur de l'histoire de la philosophie, la puissance réflexive du discours se laisse ordonner dans l'unité systématique offerte par la pragmatique. Selon ce schéma, pour qu'il y ait spéculation philosophique ou scientifique, le discours doit d'abord ménager l'ouverture réflexive permettant le développement d'une notion de vérité affranchie du référentiel. Ce premier décalage, entre la narration et ses propres virtualités interprétatives, est thématisé dans la Théogonie d'Hésiode, le plus savant des discours mythiques, comme étant la condition nécessaire d'une vérité immanente au discours. Certes, on ne peut pas attribuer au muthos le travail de conceptualisation sous sa forme achevée et proprement logique, mais on peut le présupposer dans sa fonction fondamentale, dans la mesure où ce type de discours attribue à une multiplicité donnée un ordre et un enchaînement des éléments qui la composent. Or, si la vérité noétique revendiquée par Hésiode joue un rôle important dans le développement des modèles cosmologiques présocratiques, elle n'est pas pour autant constitutive d'une modalité discursive proprement critique. Sur ce point, on conviendra qu'une rupture avec les formes traditionnelles dut présider à l'essor impressionnant que connut la philosophie milésienne dans une si courte période. Toujours est-il que la confrontation des logoi philosophiques implique un saut réflexif dont la première thématisation est l'œuvre d'un discours mythologique. Celle-ci a dû si fortement marquer les esprits qu'elle explique, en partie, le retour à l'hexamètre dactylique après l' «invention » milésienne de la prose philosophique. De fait, les premières considérations épistémologiques sur la prétention à la vérité d'un discours qui rompt avec l'immédiateté du réel seront consignées par Xénophane dans la forme poétique d'Hésiode, en se servant parfois des mêmes termes ${ }^{1}$. Comme si, par le retour au vers du discours mythique, le discours de la philosophie cherchait à consolider définitivement ce premier décalage réflexif qui est sa propre condition de possibilité.

1. Voir notamment DK 21, B34 et B35. 